\title{
PRESENTATION AND MANAGEMENT OF HEPATIC INJURY DUE TO BLUNT TRAUMA IN PATIENTS ATTENDING IN CASUALTY BLOCK OF DMCH- A STUDY OF 50 CASES
}

\author{
CHOWDHURY MMR, ${ }^{1}$ HOSSAIN SMA,${ }^{2}$ SULTANA S,${ }^{3}$ ZAMAN R, ${ }^{4}$ ALAUDDIN M ${ }^{5}$, RAHMAN A ${ }^{6}$, \\ MASUM $\mathrm{MNH}^{7}$
}

\begin{abstract}
:
Objective: To diagnose the cases of hepatic injury due to blunt abdominal trauma in a shortest possible time and find out the way of efficient and planned management of hepatic injury in our present setting.

Materials and methods: This study was done in the casualty department of Dhaka Medical College Hospital and 50 patients of hepatic injury following blunt abdominal trauma were selected over a period of January 2010 to December 2010. All 50 patients were admitted within 24 hours of incidence. The patients were diagnosed clinically by history and physical examination and relevant investigations. Immediately after admission patients were resuscitated by clearance of airway, maintenance of respiration, arrest of external bleeding and maintenance of normal circulation (ATLS Protocol). After resuscitation further management was planned depending upon the condition of the patient. Clinical presentation, overall management and outcome were evaluated by the available resources of casualty ward of DMCH.
\end{abstract}

Results: Most patients were male (88\%) and 68\% of patients were belonged to age group of 21 to 40 years. Most of the patients (90\%) were injured as a result of road traffic accidents. All patients had a history of trauma and most of them presented with abdominal pain, tenderness muscular rigidity of abdomen and shock (38\%). Only 13 (26\%) patients had isolated hepatic injury. Rest of the patients had associated other organ injuries. Majority patients (46\%) had Grade-I hepatic injury. Out of 50 patients, 46 were operated and most of them had other intra abdominal organ injuries and 4 patients were given non-operative management. Suture hepatorrhaphy was done in 38 (76\%) cases. Most common post operative complications were pulmonary in origin (24\%) and three patients were died in this series.

Conclusion: Simple technique of hemostasis such as suture hepatorrhaphy is sufficient in most cases with adequate drainage and non operative management can be tried based on haemodynamic stability.

Keywords: blunt trauma, hepatic injury, suture hepatorrhaphy.

J Dhaka Med Coll. 2016; 25(2) : 124-128

\section{Introduction:}

With the advancement of civilization the incidence of traffic accidents are also increasing and trauma represents a major public health problem. It is the principal cause of death during the first half of life $^{1}$ however fall from height and physical assault also constitute a substantial percentage of these injuries. In all trauma patients, abdominal organ injuries are quite common and liver is the second most commonly injured organ in abdominal trauma ${ }^{2,3}$ but damage to the liver is the most common cause of death after blunt abdominal injury. ${ }^{2,3}$ The most common cause of hepatic injury is blunt abdominal trauma which is secondary to motor vehicle

1. Dr. Mohammad Mahfuzur Rahman Chowdhury, Assistant professor of Urology, Dhaka Medical College, Dhaka.

2. Prof. S.M. Amjad Hossain, Professor of Surgery, Care specialized hospital, Dhaka.

3. Dr. Salma Sultana, Associate professor of Surgery, Dhaka Medical College, Dhaka.

4. Dr. Rifat Zaman, Clinical Pathologist, Dhaka Medical College Hospital, Dhaka.

5. Dr. Md. Alauddin, Medical Officer, Department of Surgery, Dhaka Medical College Hospital, Dhaka.

6. Dr. Atiar Rahman, Assistant Professor, Department of Surgery, Dhaka Medical College Hospital, Dhaka.

7. Dr. Md. Nazmul Hoque Masum, Asst. Professor, Department of Surgery, Dhaka Medical College Hospital, Dhaka. Address of correspondence: Dr. Mohammad Mahfuzur Rahman Chowdhury, Assistant Professor of Urology, Department of Urology, Dhaka Medical College, Dhaka. E-mail: mahfuz.urology@gmail.com Mobile: +8801711372737 
accidents $^{2,4}$ and is $15 \%-20 \%$ of blunt abdominal trauma. ${ }^{5}$

The mortality following hepatic injury has direct relation to the associated other organ injuries and it rises from about $20 \%$ to over $50 \%$ when four or more organs are involved. ${ }^{6}$ The present study was aimed to see different presentation and management of hepatic injury and compared with the previous study and available text references.

\section{Materials and methods:}

This prospective observational study has been conducted at the casualty department of Dhaka Medical College Hospital on 50 patients of hepatic injury following blunt abdominal trauma. All the cases were selected over a period of January 2010 to December 2010 according to schedule inclusion and exclusion criteria and they were admitted within 24 hours of incidence. The patients were diagnosed clinically by history and physical examination and relevant investigations e.g. blood grouping and cross matching, $\mathrm{Hb} \%$, USG of whole abdomen, plain X-Ray abdomen, CT scan of abdomen etc. were done and recorded. Immediately after admission patients were resuscitated by clearance of airway, maintenance of respiration, arrest of external bleeding and maintenance of normal circulation (ATLS Protocol). After resuscitation, further management was planned depending upon the condition of the patient. The cases were studied following a fixed protocol. Clinical presentation, overall management and outcome were evaluated with the available resources on casualty block, DMCH. Patient's demographics, injury details, diagnostic imaging and treatment outcome were presented in a tabulated form and the results of this study were compared with that of different recent journals of surgery and trauma.

Ethical approval: The Thesis and Dissertation Approval Committee, Bangladesh College of Physicians and Surgeons approved the study. The patients and their guardians were informed about the study, and their written informed consent were recorded.

\section{Results:}

Most patients were male (88\%) and the highest incidence $(90 \%)$ was observed in the first four decades of life and most (68\%) of them belong to age groups 21 to 40 years. Most of the patients $(90 \%)$ were injured as a result of road traffic accidents and others (10\%) were injured due to fall from height on hard blunt surface, physical assault and accidental physical trauma. All patients had a history of trauma and most of them presented with abdominal pain, tenderness muscular rigidity of abdomen and shock (38\%). Only $13(26 \%)$ patients had isolated hepatic injury. Rest of the patients had associated other organ injuries and 25(50\%) cases had intra-abdominal organ injury. Majority patients (46\%) had Grade-I hepatic injury (table-I).

Table-I

Characteristics of the cases $(n=50)$

\begin{tabular}{lc}
\hline Number of cases & $\mathrm{n}=50(100 \%)$ \\
\hline Sex & $44(88 \%)$ \\
Men & $06(12 \%)$ \\
Women & \\
Mechanism of injury & $45(90 \%)$ \\
RTA & $02(04 \%)$ \\
Fall from height & $02(04 \%)$ \\
Physical assault & $01(02 \%)$ \\
Accidental trauma & \\
Moore's grading of hepatic injury & \\
Grade I & $23(46 \%)$ \\
Grade II & $17(34 \%)$ \\
Grade III & $04(08 \%)$ \\
Grade IV & $01(02 \%)$ \\
Could not be classified & $05(10 \%)$ \\
Injury of hepatic lobe & \\
Right lobe & $37(74 \%)$ \\
Left lobe & $08(16 \%)$ \\
Both lobes & $05(10 \%)$ \\
Associated organs injury & \\
Isolated liver injury & $13(26 \%)$ \\
Multiple organs injury & $37(74 \%)$ \\
\hline
\end{tabular}


Table-II

Management of hepatic injury $(n=50)$

\begin{tabular}{llc}
\hline Procedure & Number of patient & Percentage \\
\hline a. Suture hepatorrhaphy & 38 & $76 \%$ \\
b. Resectional debridement & 02 & $04 \%$ \\
c. Use of pedicled omentum into bleeding fracture site & 02 & $04 \%$ \\
d. Laparoscopic drainage and control haemorrhage & 04 & $08 \%$ \\
e. Non-operative management & 04 & $08 \%$ \\
\hline
\end{tabular}

Out of 50 patients, 46 were operated and most of them had other intra abdominal organ injuries and 4 patients were given nonoperative management. Suture hepatorrhaphy was done in 38 (76\%) cases (table-II). Most common post operative complications were pulmonary in origin (24\%) and re-laparatomy was performed in one case due to haemorrhage. Three patients were died in this series. The average period of hospitalization was 15 days (2 to 50 days).

\section{Discussion:}

Hepatic injuries are encountered in civilian surgical practice with regular frequency. ${ }^{6}$ Diagnosis of liver injury is not always easy. Approximately 15 years ago one of the basic diagnostic procedures in injuries of parenchymatose organs of the abdominal cavity was the peritoneal lavage or surgical exploration of the abdominal cavity. Recently there has been a significant move towards sophisticated diagnostic methods with high precision, such as sonography, CT, CT angiography, MRI that, together with clinical examination are now-a-days basic methods of diagnostic algorithm. ${ }^{7,8}$ Diagnostic laparoscopy plays an important role in haemodynamically stable patient. Early diagnosis is very important in lowering the mortality from liver injury. ${ }^{9}$ Pringle in 1908 established the principle of haemorrhage control in hepatic injuries, but mortality remained high (60\%-70\%). Over the past half century mortality from hepatic injury has been reduced by early diagnosis and management. ${ }^{9}$ Mechanism of injury varies from developing to developed countries and from rural to urban area.
Linda $\mathrm{M}$ et al. ${ }^{10}$ described in his 5 years study that the patient's age ranged from 15 to 94 years (Median 26 years). Jing et al. ${ }^{11}$ showed in his experience in 348 cases of liver trauma that median age was 28 years and age range was 7 73 years. Treska V et al (2008) ${ }^{12}$ described in his study that the patient's age ranged from 2 to 70 years (Median 33.4 years). In present study age of the patients ranged from 5-65 years (median 26 years) the highest incidence $(68 \%)$ was found in persons between the age of 21-40 years. There are variations of age ranges in different corners of world as in present series but the median age and age of maximum incidence are almost same in all studies. In present series most of patients were male $(88 \%)$ and only $12 \%$ were female. Less common occurrence among females may be clue to fact that females in our country are less outgoing as chances of wounding is very limited. Zargar $\mathrm{M}$ et al. ${ }^{3}$, Jing et al. ${ }^{11}$ and Treska $\mathrm{V}$ et al. ${ }^{12}$ found male predominance in their study in different parts of world. To combat the modern civilization peoples are running fast with motor vehicles all over the world. So injury occurs due to RTA. Chong et al. ${ }^{4}$ and Zargar $\mathrm{M}$ et al. ${ }^{3}$ found the most common injury mechanism was road traffic accidents. In current study, it is $90 \%$.

Gupta SS et al. ${ }^{13}$ diagnosed liver injury in cases with blunt trauma based on physical examination along with abdominal paracentesis, serial HCT value and ultrasonography. He found $35 \%$ of patients arrived in emergency in a state of shock (40 of 105 patients). In this study, most common clinical findings were shock (38\%), abdominal pain of varying severity, tenderness of 
abdomen, rigidity and intestinal ileus. In addition to the clinical findings diagnostic abdominal tap was performed in 4 cases $(8 \%)$ and diagnostic laparoscopy 4 cases (8\%) and ultrasonography- the FAST exam(Focused Assessment with Sonography in Trauma) ${ }^{14}$ in 50 cases.

The approach to hepatic injuries management during the 30 years period after second world war remained relatively constant. Pringle time was limited to $15-20$ minutes, ${ }^{15}$ intra hepatic gauge packing was replaced by large mattress sutures to control of haemorrhage, major resection was frequently employed. Some of these procedure resulted in excessive morbidity and mortality. ${ }^{16}$ For these reasons a significant changes in therapy has occurred during the past 15-20 years. Most notable changes include non operative treatment, techniques of surgery and delayed laparoscopy to manage complications of non operative treatment. ${ }^{17}$ Extension of Pringles' time, use of hepatotomy with selective vascular ligation, insertion of omental patch into hepatotomy site or site of injury, perihepatic packing are the modifications of surgical technique. ${ }^{18}$ In present study the same incision was used as Gupta SS et al. ${ }^{13}$ showed in his publication about consecutive 105 patients management that $80 \%$ patients with liver injury could be managed by simple procedure i.e. suture hepatorrhaphy, in present study it is $76 \%$. Stone and lamb in $1975^{19}$ introduced the use of viable pedicle of omentum loosely placed into deep lobar laceration or hepatotomy site. In this series, using Pringles manoeuvre omental patch was used in $2(4 \%)$ cases. The result was excellent. Feliciano et al. ${ }^{20}$ recommended perihepatic packing in presence of oozing from raw hepatic surface or coagulopathies. He showed judicial use of perihepatic packs lead to survival rate of $60 \%$ to $90 \%$. Recent publications showed that total duration of liver packing does not increase in septic complication or bile leaks. ${ }^{18}$ In present series perihepatic packs were used in $2(4 \%)$ patients with oozing from diffuse hepatic area, Nicol et al. ${ }^{18}$ suggested to take $1^{\text {st }}$ relook laparotomy after 48 hours. In this study packs were removed after 3 days in a planned re-exploration of abdomen then drained with wide bore drain tube. Drainage after hepatic trauma has been controversial for over 80 years. Recent reports have strongly suggested that drainage is not essential in management of patients sustaining minor or modest hepatic trauma. ${ }^{1}$ In present study, wide bore drainage tubes were used as a routine. Linda et al. ${ }^{10}$ in his study on 82 cases reported pulmonary complications $20 \%$, sepsis $14 \%$. The leading complication in present study was pulmonary complication $(24 \%)$ and wound infection in $20 \%$ patients. Incidence of bile peritonitis is only $6 \%$. Three patients died in present study, all had multiple organs injury. This observation also shows that mortality following hepatic injury have direct relation to the increase number of associated organ injury. Krige $\mathrm{JE}^{21}$ had drawn similar conclusion after managing 446 patients with hepatic injury.

In recent years, the treatment of abdominal injuries has evolved and a nonoperative approach has been adopted in an increasing number of selected patients ${ }^{16}$ but there are potential risks to this strategy in terms of delayed hemorrhage necessitating laparotomy. ${ }^{22}$ Much has been written in recent journals about the role of Laparoscopy in blunt abdominal trauma. ${ }^{17}$ Most of the patients in our study underwent laparotomy. The current recommendation of non operative management based on haemodynamic stability applies to appropriately configured centers where the skills and resources necessary for emergency hepatic surgery are available. ${ }^{22}$

\section{Conclusion:}

High degree of suspicion, early diagnosis and treatment are the keys to reduce the mortality and morbidity. Simple technique of hemostasis such as suture hepatorrhaphy is sufficient in most cases with adequate drainage and nonoperative management of liver injury can be safely accomplished in haemodynamically stable patients, with the possible benefit of a shorter hospital stay.

\section{References:}

1. Mark R. Hemmila, MD \& Windy L. Wahl, MD. Management of the injured patient. In: Lawrence W. Way, Gerard M. Doherty, editors. Current Surgical Diagnosis \& Treatment $12^{\text {th }}$ edition. McGraw-Hill, 2006:207-244. 
2. Joseph DuBosea, Kenji Inabaa, Pedro G.R. Teixeiraa. Selective non-operative management of solid organ injury following blunt abdominal trauma; Injury, Feb-2007, 37:1084-1090.

3. Zargar M, Laal M. Liver Trauma: Operative and Non-operative Management. International Journal of Collaborative Research on Internal Medicine \& Public Health. 2010; Vol. 2 No. 4 :96-107

4. Chong CN, Cheung YS, Lee KF ,Rainer TH ,Lai BSP. Traumatic liver injury in Hong Kong: the management strategy and outcome. In Hong Kong Journal of Emergency Medicine. 2009; 16:208216.

5. Smith M,Driscoll P.A. Trauma. In Kirk R.M, Ribbans W.J. editors. Clinical Surgery in General RCS Course Manual. $4^{\text {th }}$ edition. London: Churchil Livingstone,UK, 2004:18-45

6. Brain R. Davidson. The liver. In: Norman S. Willams, Chirstopher J.K. Bulstrode \& P. Ronan O‘Connell, editors. Bailey \& Love's Short Practice of Surgery. $25^{\text {th }}$ edition, London:Hodder Arnold,2008:1080-1100.

7. Vyhnanek F, Denemark L, Duchac V. Basic diagnostic and therapeutic principles of liver injury. Acta Chir Orthop Traumatol Czech 2003;70: 219-225.

8. Poletti PA, Mirvis SE, Shanmuganathan K, Killeen KL, Coldwell D. CT criteria for management of blunt liver trauma: correlation with angiographic and surgical findings. Radiology 2000;216: 418427

9. David L. Dawson, and Gregory J. Jurkovich, Hepatic duct disruption from Blunt abdominal trauma; 'J Trauma; December- 1991;31: 1698-1702.

10. Linda M. Harris, Frank V. Mcl. Booth and James m. Hassett; Liver laceration-A marker of severe but sometimes subtle intraabdominal injuries in adults. Trauma, 1991;31;894-901.

11. Jing-mou Gao, Ding-yuan Du, Xing-Jizhao, Guolong liu. Liver trauma: Experience in 348 cases (1988-2000); world journal of Surgery; May-2003; $27: 703$
12. Treska V,Skalicky T,Houdek K,Smid D : Diagnosis and treatment of liver injury-the experience of the University Trauma Center. Bratisl Lek Listy 2008;109(1): 10-13

13. Gupta SS, Ahluwalia SM, Anantharam P. Liver trauma (management in 105 consecutive cases) : Journal of postgraduate medicine Dec-2001; $36: 131$

14. Moylan M; Newgard CD; Ma OJ; Sabbaj A; Rogers T; Douglass R (Oct 2007). "Association between a positive ED FAST examination and therapeutic laparotomy in normotensive blunt trauma patients". Journal of Emergency Medicine 2007;33 (3): 265-71.

15. Pringle $\mathrm{JH}$; "Notes on the arrest of hepatic hemorrhage due to trauma”, Ann. Surg. 1908; 48; 541-9

16. Brammer R.D, Bramhall S.R, Mirza D.F, Mayer $\mathrm{AD}$, McMaster P and Buckles J.A.C ;A 10 years experience of complex liver trauma.BJS 2002:89; 1532-1537.

17. Pilgrim, Charlestt, Usatoff, Val. Role of Laparoscopy in blunt liver trauma. ANZ journal of surgery May 2006; 76(5): 403-406.

18. Nicol A.J, Hommes M, Primrose R, Navsara P.H. and Krige JE. Packing for control of haamorahage in major liver trauma. World journal of Surgery 2007; 31:569-574.

19. Stone HH, Lamb JM. Use of Pedicled omentum as an autogenous pack for control of haemorrage in major injuries of the liver. Surg Gynaecol \& obstet 1975; 141: 92-94

20. Faliciano V, Kenneth L. Mattoxjon M. Burch, et al, 'Packing for control of hepatic hemorrhage, Trauma; Aug -1986;26; 738-743

21. Krige JE, Bornman PC, Terblanche J. Liver Trauma in 446 Patients. , S. Afr J Surg 1997: 35:10-15

22. Holland MJ, Little JM. Expanding role of non operative management in patients with blunt liver injury. BJS 2004; 91 (3): 317-321. 